PROCEEDINGS OF THE

AMERICAN MATHEMATICAL SOCIETY

Volume 128, Number 11, Pages 3159-3166

S 0002-9939(00)05445-9

Article electronically published on May 18, 2000

\title{
QUADRATIC DIVISION ALGEBRAS REVISITED (REMARKS ON AN ARTICLE BY J. M. OSBORN)
}

\author{
ERNST DIETERICH
}

(Communicated by Lance W. Small)

\begin{abstract}
In his remarkable article "Quadratic division algebras" (Trans. Amer. Math. Soc. 105 (1962), 202-221), J. M. Osborn claims to solve 'the problem of determining all quadratic division algebras of order 4 over an arbitrary field $F$ of characteristic not two ... modulo the theory of quadratic forms over $F^{\prime}$ (cf. p. 206). While we shall explain in which respect he has not achieved this goal, we shall on the other hand complete Osborn's basic results (by a reasoning which is finer than his) to derive in the real ground field case a classification of all 4-dimensional quadratic division algebras and the construction of a 49-parameter family of pairwise nonisomorphic 8-dimensional quadratic division algebras.

To make these points clear, we begin by reformulating Osborn's fundamental observations on quadratic algebras in categorical terms.
\end{abstract}

\section{First equivalence for anisotropic Quadratic algebras}

Let $k$ be a field of characteristic not two. An algebra is a $k$-vectorspace $A$ endowed with a $k$-bilinear multiplication $A \times A \rightarrow A,(x, y) \mapsto x y$. A nonzero algebra $A$ is called quadratic in case an identity element $1 \in A$ exists and each $x \in A$ satisfies an equation $x^{2}=\alpha x+\beta 1$ with $\alpha, \beta \in k$.

Given any quadratic algebra $A$, Frobenius' lemma states that the set $V=\{v \in$ $\left.A \mid v^{2} \in k 1\right\} \backslash(k 1 \backslash\{0\})$ of purely imaginary elements of $A$ forms a linear subspace of $A$ which is supplementary to $k 1$ (cf. [9, [3], 11]). Accordingly, each $x \in A$ has unique decomposition $x=\lambda(x) 1+\iota(x)$, with $\lambda(x) \in k$ and $\iota(x) \in V$. The linear form $\lambda: A \rightarrow k$ gives rise to the bilinear form ()$: V \times V \rightarrow k,(x, y)=-\lambda(x y)$, and the projection $\iota: A \rightarrow V$ gives rise to the bilinear multiplication $\circ: V \times V \rightarrow$ $V, v \circ w=\iota(v w)$ which by Frobenius' lemma is anticommutative.

Let us introduce the category $\mathcal{A}^{q}$ of all quadratic $k$-algebras, and the category $\mathcal{A}^{a b}$ of all anticommutative $k$-algebras endowed with a bilinear form. Morphisms in $\mathcal{A}^{q}$ are algebra morphisms respecting identity elements, while morphisms in $\mathcal{A}^{a b}$ are algebra morphisms respecting bilinear forms. In these terms, Frobenius' lemma gives rise to the map of object classes

$$
\Phi: O b\left(\mathcal{A}^{q}\right) \rightarrow O b\left(\mathcal{A}^{a b}\right), \Phi(A)=(V, \circ,()) .
$$

Received by the editors December 8, 1998 and, in revised form, January 4, 1999.

2000 Mathematics Subject Classification. Primary 17A35, 17A45, 57S25.

(C)2000 American Mathematical Society 
Each morphism of quadratic algebras $\mu: A \rightarrow A^{\prime}$ is of the form $\mu=\left(\begin{array}{cc}i d & \epsilon \\ 0 & \nu\end{array}\right)$, where $i d: k 1 \stackrel{\sim}{\rightarrow} k 1^{\prime}, 0: k 1 \rightarrow V^{\prime}, \epsilon: V \rightarrow k 1^{\prime}$ and $\nu: V \rightarrow V^{\prime}$ are its linear components with respect to the Frobenius decomposition of $A$ and $A^{\prime}$ respectively. The hope that $\nu$ might even be a morphism $\Phi(A) \rightarrow \Phi\left(A^{\prime}\right)$ in $\mathcal{A}^{a b}$ is disappointed by considering the example $A=A^{\prime}=k[X] /\left(X^{2}-1\right), \mu(\bar{X})=\overline{1}$. But still, the following facts are readily verified:

(1) If $\epsilon=0$, then $\nu \in \mathcal{A}^{a b}\left(\Phi(A), \Phi\left(A^{\prime}\right)\right)$.

(2) If $\mu$ is an isomorphism, then $\epsilon=0$.

(3) If the quadratic form $q_{A}: A \rightarrow k, q_{A}(x)=\lambda(x)^{2}+(\iota(x), \iota(x))$ is anisotropic, then $\epsilon=0$.

Looking in the converse direction, there is indeed a functor $\mathcal{F}: \mathcal{A}^{a b} \rightarrow \mathcal{A}^{q}$, defined on objects by $\mathcal{F}(V, \circ,())=k \times V$ with multiplication $(\alpha, v)(\beta, w)=$ $(\alpha \beta-(v, w), \alpha w+\beta v+v \circ w)$, and on morphisms by $\mathcal{F}(\nu)=\mathbb{I}_{k} \times \nu$. By construction, $\Phi$ and $\mathcal{F}$ are related by

(4) $\mathcal{F} \Phi(A) \stackrel{\sim}{\rightarrow} A$ for all $A \in \mathcal{A}^{q}$.

The hope that $\mathcal{F}$ might even be an equivalence of categories is disappointed by comparing the endomorphism monoids of the objects $B=(k, \circ,(-1)) \in \mathcal{A}^{a b}$ and $A=\mathcal{F}(B) \stackrel{\sim}{\rightarrow} k[X] /\left(X^{2}-1\right)$ respectively: $\mathcal{A}^{a b}(B, B)=\left\{\mathbb{I}_{k},-\mathbb{I}_{k}\right\}$, while $\mathcal{A}^{q}(A, A)$ has cardinality 4 . But still, the functor $\mathcal{F}$ is 'nearly an equivalence' in the following sense.

Proposition 1. The functor $\mathcal{F}: \mathcal{A}^{a b} \rightarrow \mathcal{A}^{q}$ has the following properties:

(i) It is faithful and dense. It detects and exhausts isomorphisms 1

(ii) It induces a bijection $\overline{\mathcal{F}}: O b\left(\mathcal{A}^{a b}\right) / \simeq \stackrel{\sim}{\rightarrow} O b\left(\mathcal{A}^{q}\right) / \simeq$ between the sets of isoclasses of the respective categories.

(iii) It induces an equivalence of full subcategories $\mathcal{F}^{a}: \mathcal{A}^{a b a} \stackrel{\sim}{\rightarrow} \mathcal{A}^{q a}$, where $\mathcal{A}^{a b a}$ is formed by all objects $B=(V, \circ,())$ such that the quadratic form $q_{B}: k \times V \rightarrow k$, $q_{B}(\alpha, v)=\alpha^{2}+(v, v)$ is anisotropic, and $\mathcal{A}^{q a}$ is formed by all quadratic algebras $A$ whose quadratic form $q_{A}: A \rightarrow k, q_{A}(x)=\lambda(x)^{2}+(\iota(x), \iota(x))$ is anisotropic. $A$ quasi-inverse functor $\Phi^{a}: \mathcal{A}^{q a} \stackrel{\sim}{\rightarrow} \mathcal{A}^{a b a}$ to $\mathcal{F}^{a}$ is given by $\Phi^{a}(A)=\Phi(A)$ and $\Phi^{a}(\mu)=\nu$.

Using (1)-(4), the proof of Proposition 1 amounts to a routine verification. The fact that the map of object classes $\mathcal{F}: O b\left(\mathcal{A}^{a b}\right) \rightarrow O b\left(\mathcal{A}^{q}\right)$ is well-defined and satisfies (4) constitutes [14], Theorem 1.

Let us denote by $\mathcal{A}^{q 0}$ the full subcategory of $\mathcal{A}^{q}$ formed by all quadratic algebras without zero divisors. It is easily seen that $\mathcal{A}^{q 0} \subset \mathcal{A}^{q a}$. Thus the equivalence $\Phi^{a}: \mathcal{A}^{q a} \stackrel{\sim}{\rightarrow} \mathcal{A}^{a b a}$ induces an equivalence $\Phi^{0}: \mathcal{A}^{q 0} \stackrel{\sim}{\rightarrow} \mathcal{A}^{a b 0}$, where $\mathcal{A}^{a b 0}$ is the full subcategory of $\mathcal{A}^{a b a}$ formed by all $B \in \mathcal{A}^{a b a}$ such that $\mathcal{F} B \in \mathcal{A}^{q 0}$. The interesting question how to characterize the subclass $\mathcal{A}^{a b 0} \subset \mathcal{A}^{a b}$ internally is answered in [14], Theorem 2 and Theorem 3 as follows.

Proposition 2. An object $B=(V, \circ,())$ in $\mathcal{A}^{a b}$ belongs to the subclass $\mathcal{A}^{a b 0}$ if and only if it satisfies the following two conditions:

(a) The quadratic form $q_{B}: k \times V \rightarrow k, q_{B}(\alpha, v)=\alpha^{2}+(v, v)$ is anisotropic.

(b) If $v, w \in V$ are linearly independent, then $v, w, v \circ w$ are also.

\footnotetext{
${ }^{1}$ The functor $\mathcal{F}: \mathcal{A}^{a b} \rightarrow \mathcal{A}^{q}$ is said to detect isomorphisms in case for all $B, B^{\prime} \in \mathcal{A}^{a b}$ and for all $\nu \in \mathcal{A}^{a b}\left(B, B^{\prime}\right)$, if $\mathcal{F}(\nu)$ is an isomorphism, then $\nu$ is also. We say that $\mathcal{F}: \mathcal{A}^{a b} \rightarrow \mathcal{A}^{q}$ exhausts isomorphisms in case for all $B, B^{\prime} \in \mathcal{A}^{a b}$ and for each isomorphism $\mu \in \mathcal{A}^{q}\left(\mathcal{F} B, \mathcal{F} B^{\prime}\right)$ there exists a morphism $\nu \in \mathcal{A}^{a b}\left(B, B^{\prime}\right)$ such that $\mathcal{F}(\nu)=\mu$.
} 
At this stage, turning to the problem of determining all 4-dimensional quadratic division algebras, Osborn proceeds (p. 206 ff.) to observe that it 'breaks' (sic!) into the two distinct problems of finding all bilinear forms in three variables satisfying (a) and of finding all 3-dimensional anticommutative algebras satisfying (b). Concerning the first of these, he is content with the remark that it already belongs to the theory of quadratic forms over $k$. Concerning the second, he indeed succeeds in reducing the classification problem for the algebras in question to a problem in the theory of quadratic forms over $k$ (Theorem 5). However, in doing so he considers classification up to algebra isomorphism, and not up to isomorphism in $\mathcal{A}^{a b}$, i.e. orthogonal algebra isomorphism. Thus, regarding quadratic division algebras, he handles the second subproblem in a category with too many morphisms! Consequently, interesting as Osborn's Theorem 5 and Theorem 8 (dealing with 7-dimensional anticommutative algebras) are as such, they convey but little information on the classification of quadratic division algebras. The degree of inaccuracy arising in this way can be made explicit in the classical case $k=\mathbb{R}$. Here, the 3-dimensional anticommutative algebras satisfying (b) are completely classified up to algebra isomorphism by a single 1-parameter family ([14, Theorem 6), whereas there is a 9-parameter family of pairwise nonisomorphic 3-dimensional objects in $\mathcal{A}^{a b 0}$ (cf. section 3 ).

This finishes our discussion of Osborn's article. We shall proceed by analyzing the category $\mathcal{A}^{a b 0}$ so closely as to obtain, in the real ground field case, a classification of all 4-dimensional quadratic division algebras and the construction of a 49-parameter family of pairwise nonisomorphic 8-dimensional quadratic division algebras. It turns out that this approach, based on Proposition 1 and Proposition 2 , reveals a conceptual view of the material discussed in [4]-[7], generalizing it considerably and provoking further investigations.

\section{SeCond Equivalence For QuAdratiC ANisotropiC Algebras}

Let us consider the category $\mathcal{E}$ whose objects are triples $(V, \xi, \eta)$ consisting of a symmetric bilinear $k$-space 2 $V=(V,\langle\rangle)$ and linear maps $\xi: V \wedge V \rightarrow k$ and $\eta: V \wedge V \rightarrow V$. Morphisms $\nu:(V, \xi, \eta) \rightarrow\left(V^{\prime}, \xi^{\prime}, \eta^{\prime}\right)$ in $\mathcal{E}$ are orthogonal linear maps $\nu: V \rightarrow V^{\prime}$ satisfying $\xi=\xi^{\prime}(\nu \wedge \nu)$ and $\nu \eta=\eta^{\prime}(\nu \wedge \nu)$. An obvious isomorphism of categories $\mathcal{A}^{a b} \stackrel{\tilde{\rightarrow}}{\rightarrow} \mathcal{E},(V, \circ,()) \mapsto((V,\langle\rangle), \xi, \eta), \nu \mapsto \nu$ is obtained on decomposing the bilinear form ( ) into its symmetric part $\langle v, w\rangle=\frac{1}{2}((v, w)+(w, v))$ and its antisymmetric part $-\xi(v \wedge w)=\frac{1}{2}((v, w)-(w, v))$, and on setting $\eta(v \wedge w)=v \circ w$. Composing the inverse of this isomorphism with $\mathcal{F}: \mathcal{A}^{a b} \rightarrow \mathcal{A}^{q}$, we obtain the functor $\mathcal{G}: \mathcal{E} \rightarrow \mathcal{A}^{q}$. In explicit terms, $\mathcal{G}$ is given on objects by $\mathcal{G}((V,\langle\rangle), \xi, \eta)=$ $k \times V$ with multiplication

$$
(\alpha, v)(\beta, w)=(\alpha \beta-\langle v, w\rangle+\xi(v \wedge w), \alpha w+\beta v+\eta(v \wedge w))
$$

and on morphisms by $\mathcal{G}(\nu)=\mathbb{I}_{k} \times \nu$. Transferring the results of the previous section

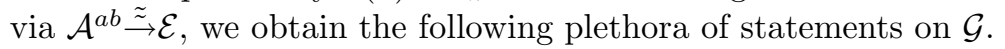

Theorem 3. The functor $\mathcal{G}: \mathcal{E} \rightarrow \mathcal{A}^{q}$ has the following properties:

(i) It is faithful and dense. It detects and exhausts isomorphisms.

\footnotetext{
${ }^{2} \mathrm{~A}$ symmetric bilinear $k$-space $(V,\langle\rangle)$ is a $k$-vectorspace $V$ endowed with a symmetric $k$ bilinear form \langle\rangle$: V \times V \rightarrow k$. A morphism $\nu:(V,\langle\rangle) \rightarrow\left(V^{\prime},\langle\rangle^{\prime}\right)$ of symmetric bilinear $k$-spaces is an orthogonal linear map, i.e. a linear map $\nu: V \rightarrow V^{\prime}$ satisfying $\langle v, w\rangle=\langle\nu(v), \nu(w)\rangle^{\prime}$ for all $v, w \in V$.
} 
(ii) It induces a bijection $\overline{\mathcal{G}}: O b(\mathcal{E}) / \simeq \stackrel{\sim}{\rightarrow} O b\left(\mathcal{A}^{q}\right) / \simeq$ between the sets of isoclasses of the respective categories.

(iii) It induces an equivalence of full subcategories $\mathcal{G}^{a}: \mathcal{E}^{a} \stackrel{\sim}{\rightarrow} \mathcal{A}^{q a}$, where $\mathcal{E}^{a}$ is formed by all objects $E=((V,\langle\rangle), \xi, \eta)$ such that the quadratic form $q_{E}: k \times V \rightarrow$ $k, q_{E}(\alpha, v)=\alpha^{2}+\langle v, v\rangle$ is anisotropic. A quasi-inverse functor $\Gamma^{a}: \mathcal{A}^{q a} \stackrel{\sim}{\rightarrow} \mathcal{E}^{a}$ to $\mathcal{G}^{a}$ is given by $\Gamma^{a}(A)=((V,\langle\rangle), \xi, \eta)$ and $\Gamma^{a}(\mu)=\nu$, where $V$ is the hyperplane of purely imaginary elements in $A,\langle v, w\rangle=-\frac{1}{2} \lambda(v w+w v), \xi(v \wedge w)=$ $\frac{1}{2} \lambda(v w-w v), \eta(v \wedge w)=\iota(v w)$ and $\mu=i d \oplus \nu$.

(iv) It induces an equivalence of full subcategories $\mathcal{G}^{0}: \mathcal{E}^{0} \stackrel{\sim}{\rightarrow} \mathcal{A}^{q 0}$, where $\mathcal{E}^{0}$ is formed by all objects $E=((V,\langle\rangle), \xi, \eta)$ satisfying the following two conditions:

(a) The quadratic form $q_{E}: k \times V \rightarrow k, q_{E}(\alpha, v)=\alpha^{2}+\langle v, v\rangle$ is anisotropic.

(b) If $v, w \in V$ are linearly independent, then $v, w, \eta(v \wedge w)$ are also.

The functor $\Gamma^{0}: \mathcal{A}^{q 0} \stackrel{\sim}{\rightarrow} \mathcal{E}^{0}$ induced by $\Gamma^{a}: \mathcal{A}^{q a} \stackrel{\sim}{\rightarrow} \mathcal{E}^{a}$ is quasi-inverse to $\mathcal{G}^{0}$.

Given any category $\mathcal{Z}$ for which a function $\operatorname{dim}: O b(\mathcal{Z}) \rightarrow \mathbb{N} \cup\{\infty\}$ is defined, we denote for any $n \in \mathbb{N}$ by $\mathcal{Z}_{n}$ the full subcategory of $\mathcal{Z}$ formed by $\operatorname{dim}^{-1}(n)$. Thus the equivalence $\Gamma^{0}: \mathcal{A}^{q 0} \stackrel{\sim}{\rightarrow} \mathcal{E}^{0}$ induces for each $n \in \mathbb{N} \backslash\{0\}$ an equivalence $\Gamma_{n}^{0}: \mathcal{A}_{n}^{q 0} \stackrel{\sim}{\rightarrow} \mathcal{E}_{n-1}^{0}$. Moreover, $\mathcal{A}_{n}^{q 0}$ coincides with the category of all $n$-dimensional quadratic division algebras 3

\section{DisSIDENT MORPHISMS}

Henceforth let $k=\mathbb{R}$. Applying Theorem 3 (iv), we aim at classifying all $n$-dimensional real quadratic division algebras by way of classifying all triples $((V,\langle\rangle), \xi, \eta) \in \mathcal{E}_{n-1}^{0}$. By Sylvester's inertia theorem, property (a) is equivalent to \langle\rangle being positive definite.

Therefore, when forgetting about $\xi$, we are left with the problem to describe all dissident morphisms, i.e. all linear maps $\eta: V \wedge V \rightarrow V$ satisfying (b), where $V=(V,\langle\rangle)$ is a euclidian vectorspace. Combining Theorem 3 (ii) and the celebrated $(1,2,4,8)$-Theorem $([10$, , 13], 2], [1] $)$, we recognize at once that dissident morphisms exist in the exceptional dimensions $0,1,3,7$ only.

Corollary 4. If $\eta: V \wedge V \rightarrow V$ is a dissident morphism, then $\operatorname{dim}_{\mathbb{R}} V \in\{0,1,3,7\}$.

Proof. From any dissident morphism $\eta: V \wedge V \rightarrow V$ we may form the objects $(V, o, \eta) \in \mathcal{E}^{0}$ and $A=\mathcal{G}^{0}(V, o, \eta) \in \mathcal{A}^{q 0}$. Application of the (1,2,4,8)-Theorem to $A$ yields the assertion.

Conversely, dissident morphisms in fact do exist in each of the dimensions $0,1,3,7$. Namely, each vectorproduct $\pi: V \wedge V \rightarrow V$ provides a classical example of a dissident morphism. And indeed, vectorproducts are known ([8], [12]) to constitute 4 isoclasses, represented by those $\pi: V \wedge V \rightarrow V$ which arise from the alternative division algebras $A \in\{\mathbb{R}, \mathbb{C}, \mathbb{H}, \mathbb{O}\}$ via $\Gamma^{0}(A)=((V,\langle\rangle), o, \pi)$. Moreover, apart from providing the simplest examples of dissident morphisms, vectorproducts even serve to produce new dissident morphisms via composition with definite endomorphisms 5

\footnotetext{
${ }^{3}$ An algebra $A$ is called division algebra in case the linear endomorphisms $a$ ? : $A \rightarrow A, x \mapsto a x$ and $? a: A \rightarrow A, x \mapsto x a$ are bijective for all $a \in A \backslash\{0\}$.

${ }^{4}$ A linear map $\pi: V \wedge V \rightarrow V$, defined for a euclidian vectorspace $V$, is called a vectorproduct in case $\langle\pi(u \wedge v), w\rangle=\langle u, \pi(v \wedge w)\rangle$ and $|\pi(u \wedge v)|^{2}=|u|^{2}|v|^{2}-\langle u, v\rangle^{2}$ for all $u, v, w \in V$.

${ }^{5}$ A linear endomorphism $\varepsilon: V \rightarrow V$ of a euclidian vectorspace $V$ is called definite in case the quadratic form $q_{\varepsilon}: V \rightarrow \mathbb{R}, q_{\varepsilon}(v)=\langle v, \varepsilon(v)\rangle$ is positive definite or negative definite.
} 
Lemma 5. Let $\pi: V \wedge V \rightarrow V$ be a vectorproduct.

(i) If $\varepsilon: V \rightarrow V$ is a definite endomorphism, then $\varepsilon \pi: V \wedge V \rightarrow V$ is a dissident morphism.

(ii) If $\eta: V \wedge V \rightarrow V$ is a dissident morphism and $\operatorname{dim}_{\mathbb{R}} V \leq 3$, then there exists a definite endomorphism $\varepsilon: V \rightarrow V$ such that $\eta=\varepsilon \pi$.

Proof. (i) Let $v, w \in V$ be nonproportional. The vectorproduct property of $\pi$ implies $\mathbb{R} v \oplus \mathbb{R} w \subset \pi(v \wedge w)^{\perp}$ and $\pi(v \wedge w) \neq 0$. Definiteness of $\varepsilon$ further implies $\langle\pi(v \wedge w), \varepsilon \pi(v \wedge w)\rangle \neq 0$, whence $\varepsilon \pi(v \wedge w) \notin \mathbb{R} v \oplus \mathbb{R} w$.

(ii) In case $\operatorname{dim}_{\mathbb{R}} V \leq 1$, the assertion is trivially true. If $\operatorname{dim}_{\mathbb{R}} V=3$, then $\pi$ is an isomorphism. Given $u \in V \backslash\{0\}$, choose $v, w \in u^{\perp}$ such that $u=\pi(v \wedge w)$. Then $\eta(v \wedge w) \notin \mathbb{R} v \oplus \mathbb{R} w=u^{\perp}$. Therefore $\left\langle u, \eta \pi^{-1}(u)\right\rangle=\langle u, \eta(v \wedge w)\rangle \neq 0$. Hence $\varepsilon=\eta \pi^{-1}$ is definite and $\varepsilon \pi=\eta$.

It will be useful to know that any nonzero dissident morphism of the form $\eta=\varepsilon \pi$, with $\varepsilon$ and $\pi$ as in Lemma 5, determines its factors $\varepsilon$ and $\pi$ uniquely up to sign. Heading for this result (cf. Proposition 7 below) let us first consider an arbitrary given dissident morphism $\eta: V \wedge V \rightarrow V$. We briefly write $v w=\eta(v \wedge w)$. Then for each $v \in V \backslash\{0\}$, the endomorphism $v ?: V \rightarrow V$ induces an epimorphism $v^{\perp} \rightarrow v v^{\perp}$ which (by dissidence) in fact is an isomorphism. Thus $v v^{\perp}$ is a hyperplane in $V$. Hence $\eta$ determines a selfmap $\eta_{\mathbb{P}}: \mathbb{P}(V) \rightarrow \mathbb{P}(V)$, defined by $\eta_{\mathbb{P}}(\mathbb{R} v)=\left(v v^{\perp}\right)^{\perp}$. Observe that $\pi_{\mathbb{P}}=\mathbb{I}_{\mathbb{P}(V)}$ for each vectorproduct $\pi: V \wedge V \rightarrow V$.

The adjoint of an endomorphism $\varepsilon: V \rightarrow V$ will be denoted by $\varepsilon^{*}: V \rightarrow V$. If $\varepsilon$ is invertible, then so is $\varepsilon^{*}$ and $\left(\varepsilon^{*}\right)^{-1}=\left(\varepsilon^{-1}\right)^{*}$ will be denoted by $\varepsilon^{-*}$.

Lemma 6. Let $\eta=\varepsilon \pi$, where $\varepsilon: V \rightarrow V$ is a definite endomorphism and $\pi$ : $V \wedge V \rightarrow V$ is a vectorproduct. Then $\eta_{\mathbb{P}}=\mathbb{P}\left(\varepsilon^{-*}\right)$.

Proof. Let $v, \bar{v} \in V \backslash\{0\}$ such that $\eta_{\mathbb{P}}(\mathbb{R} v)=\mathbb{R} \bar{v}$. Then

$$
\left\langle\varepsilon^{*}(\bar{v}), \pi(v \wedge x)\right\rangle=\langle\bar{v}, \varepsilon \pi(v \wedge x)\rangle=\langle\bar{v}, v x\rangle=0
$$

holds for all $x \in v^{\perp}$. Thus $\mathbb{R} \varepsilon^{*}(\bar{v})=\pi_{\mathbb{P}}(\mathbb{R} v)=\mathbb{R} v$, whence $\eta_{\mathbb{P}}(\mathbb{R} v)=\mathbb{R} \bar{v}=$ $\mathbb{P}\left(\varepsilon^{-*}\right)(\mathbb{R} v)$.

Proposition 7. Let $\eta=\varepsilon \pi=\varepsilon^{\prime} \pi^{\prime}$, where $\varepsilon, \varepsilon^{\prime}: V \rightarrow V$ are definite endomorphisms and $\pi, \pi^{\prime}: V \wedge V \rightarrow V$ are nonzero vectorproducts. Then either $(\varepsilon, \pi)=\left(\varepsilon^{\prime}, \pi^{\prime}\right)$ or $(\varepsilon, \pi)=\left(-\varepsilon^{\prime},-\pi^{\prime}\right)$.

Proof. The hypothesis implies by Lemma 6 that $\eta_{\mathbb{P}}=\mathbb{P}\left(\varepsilon^{-*}\right)=\mathbb{P}\left(\varepsilon^{\prime-*}\right)$. In view of the short exact sequence

$$
1 \longrightarrow G L(\mathbb{R}) \longrightarrow G L(V) \longrightarrow P G L(V) \longrightarrow 1
$$

we conclude that $\varepsilon=r \varepsilon^{\prime}$ for some $r \in \mathbb{R} \backslash\{0\}$. Hence we obtain $r \varepsilon^{\prime} \pi=\varepsilon^{\prime} \pi^{\prime}$, whence $r \pi=\pi^{\prime}$. Since both $\pi$ and $\pi^{\prime}$ are nonzero vectorproducts, we infer that $r=1$ or $r=-1$.

\section{The matrix Pair Problem $\left(\mathbb{R}_{\text {ant }}^{m \times m} \times \mathbb{R}_{\text {pos }}^{m \times m}\right) / O_{\pi}\left(\mathbb{R}^{m}\right)$}

Let us return to the equivalent categories $\mathcal{A}_{n}^{q 0} \sim_{\rightarrow} \mathcal{E}_{n-1}^{0}$ in question. We know that they are nonempty if and only if $n \in\{1,2,4,8\}$. Therefore let $m \in\{0,1,3,7\}$ and $n=m+1$, henceforth.

Choose a vectorproduct $\pi: \mathbb{R}^{m} \wedge \mathbb{R}^{m} \rightarrow \mathbb{R}^{m}$ with respect to the natural scalar product $v \bullet w=v^{T} w$. The orthogonal group $O\left(\mathbb{R}^{m}\right)$ acts canonically on the set 
of all vectorproducts for $\mathbb{R}^{m}$ via $\sigma \cdot \pi^{\prime}=\sigma \pi^{\prime}\left(\sigma^{-1} \wedge \sigma^{-1}\right)$. Denote by $O_{\pi}\left(\mathbb{R}^{m}\right)=$ $\left\{\sigma \in O\left(\mathbb{R}^{m}\right) \mid \sigma \cdot \pi=\pi\right\}$ the isotropy group of $\pi$ in $O\left(\mathbb{R}^{m}\right)$. Moreover, denote by $\mathbb{R}_{\text {ant }}^{m \times m}$ (resp. $\mathbb{R}_{\text {pos }}^{m \times m}$ ) the set of all antisymmetric (resp. positive definite) matrices in $\mathbb{R}^{m \times m}$, and set $\mathcal{P}_{m}=\mathbb{R}_{\text {ant }}^{m \times m} \times \mathbb{R}_{\text {pos }}^{m \times m}$. Then the map $\Lambda_{\pi}: \mathcal{P}_{m} \rightarrow \mathcal{A}_{n}^{q},(X, Y) \mapsto$ $\mathcal{G}\left(\mathbb{R}^{m}, \xi, \eta\right)$, where $\xi(v \wedge w)=v^{T} X w$ and $\eta(v \wedge w)=Y \pi(v \wedge w)$, has the following properties.

Theorem 8. (i) For each matrix pair $(X, Y) \in \mathcal{P}_{m}$ the n-dimensional quadratic algebra $\Lambda_{\pi}(X, Y)$ is a division algebra.

(ii) For each quadratic division algebra $A$ of dimension $n \leq 4$ there exists a matrix pair $(X, Y) \in \mathcal{P}_{m}$ such that $\Lambda_{\pi}(X, Y) \stackrel{\sim}{\rightarrow} A$.

(iii) For all matrix pairs $(X, Y),\left(X^{\prime}, Y^{\prime}\right) \in \mathcal{P}_{m}$ of size $m>1$ the quadratic division algebras $\Lambda_{\pi}(X, Y)$ and $\Lambda_{\pi}\left(X^{\prime}, Y^{\prime}\right)$ are isomorphic if and only if $\left(S X S^{T}, S Y S^{T}\right)$ $=\left(X^{\prime}, Y^{\prime}\right)$ for some $S \in O_{\pi}\left(\mathbb{R}^{m}\right)$.

Proof. (i) and (ii) follow from Theorem 3, Lemma 5 and the fact that $-\mathbb{I}_{V}$ : $(V, \xi, \eta) \stackrel{\sim}{\rightarrow}(V, \xi,-\eta)$ is an isomorphism for each triple $(V, \xi, \eta) \in \mathcal{E}$.

(iii) Let $(X, Y),\left(X^{\prime}, Y^{\prime}\right) \in \mathcal{P}_{m}$ be given. If $\left(S X S^{T}, S Y S^{T}\right)=\left(X^{\prime}, Y^{\prime}\right)$ for some $S \in O_{\pi}\left(\mathbb{R}^{m}\right)$, then the orthogonal automorphism $\sigma: \mathbb{R}^{m} \stackrel{\sim}{\rightarrow} \mathbb{R}^{m}$ corresponding to $S$ is an isomorphism $\sigma:\left(\mathbb{R}^{m}, \xi, \eta\right) \stackrel{\sim}{\rightarrow}\left(\mathbb{R}^{m}, \xi^{\prime}, \eta^{\prime}\right)$ in $\mathcal{E}$. Accordingly $\mathbb{I}_{\mathbb{R}} \times \sigma$ :

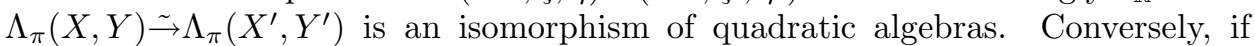
$\varphi: \Lambda_{\pi}(X, Y) \stackrel{\sim}{\rightarrow} \Lambda_{\pi}\left(X^{\prime}, Y^{\prime}\right)$ is an isomorphism of quadratic algebras, then $\varphi=$ $\mathbb{I}_{\mathbb{R}} \times \sigma$ for some isomorphism $\sigma:\left(\mathbb{R}^{m}, \xi, \eta\right) \stackrel{\sim}{\rightarrow}\left(\mathbb{R}^{m}, \xi^{\prime}, \eta^{\prime}\right)$ in $\mathcal{E}$. Hence the matrix $S \in O\left(\mathbb{R}^{m}\right)$ corresponding to $\sigma$ satisfies $S X S^{T}=X^{\prime}$ and, due to Proposition 7 , both $S Y S^{T}=Y^{\prime}$ and $S \in O_{\pi}\left(\mathbb{R}^{m}\right)$.

Let us draw conclusions for the classification of $\mathcal{A}_{n}^{q 0}$. If $m \leq 1$, then $\mathcal{E}_{m}^{0}$ consists of one isoclass represented by $\left(\mathbb{R}^{m}, o, o\right)$. Accordingly $\mathcal{A}_{n}^{q 0}$ consists of one isoclass, represented by $\mathbb{R}$ and $\mathbb{C}$ respectively.

If $m=3$, then $O_{\pi}\left(\mathbb{R}^{3}\right)=S O\left(\mathbb{R}^{3}\right)$. Accordingly, Theorem 8 yields the following

Corollary 9. The map $\Lambda_{\pi}: \mathcal{P}_{3} \rightarrow \mathcal{A}_{4}^{q 0}$ induces a bijection

$$
\bar{\Lambda}_{\pi}: \mathcal{P}_{3} / S O\left(\mathbb{R}^{3}\right) \stackrel{\sim}{\rightarrow} \mathcal{A}_{4}^{q 0} / \simeq \text {. }
$$

In order to classify the $S O\left(\mathbb{R}^{3}\right)$-orbits of the matrix pair set $\mathcal{P}_{3}$, we introduce the set $\mathcal{K}_{3}=\mathbb{R}^{3} \times \mathbb{R}^{3} \times \mathcal{T}_{3}$ where $\mathcal{T}_{3}=\left\{\delta \in \mathbb{R}^{3} \mid 0<\delta_{1} \leq \delta_{2} \leq \delta_{3}\right\}$, and we define the map $\Psi: \mathcal{K}_{3} \rightarrow \mathcal{P}_{3}$ by $\Psi(x, y, \delta)=\left(M_{x}, M_{y}+\Delta_{\delta}\right)$ where

$$
M_{x}=\left(\begin{array}{rrr}
0 & -x_{3} & x_{2} \\
x_{3} & 0 & -x_{1} \\
-x_{2} & x_{1} & 0
\end{array}\right) \quad \text { and } \quad \Delta_{\delta}=\left(\begin{array}{lll}
\delta_{1} & 0 & 0 \\
0 & \delta_{2} & 0 \\
0 & 0 & \delta_{3}
\end{array}\right) .
$$

Moreover we denote by $S O_{\delta}\left(\mathbb{R}^{3}\right)=\left\{S \in S O\left(\mathbb{R}^{3}\right) \mid S \Delta_{\delta} S^{T}=\Delta_{\delta}\right\}$ the isotropy group of $\Delta_{\delta}$ in $S O\left(\mathbb{R}^{3}\right)$, for any $\delta \in \mathcal{T}_{3}$.

If now $(X, Y) \in \mathcal{P}_{3}$ is given, decompose $Y=Y^{a}+Y^{s}$ into its antisymmetric summand $Y^{a}$ and its symmetric summand $Y^{s}$, transform $Y^{s}$ into $\Delta_{\delta}$ applying Jacobis spectral theorem, and observe that $S M_{x} S^{T}=M_{S x}$ for all $(S, x) \in S O\left(\mathbb{R}^{3}\right) \times$ $\mathbb{R}^{3}$, to prove

Lemma 10. The map $\Psi: \mathcal{K}_{3} \rightarrow \mathcal{P}_{3}$ induces a bijection $\bar{\Psi}: \mathcal{K}_{3} / \sim \stackrel{\sim}{\rightarrow} \mathcal{P}_{3} / S O\left(\mathbb{R}^{3}\right)$, where $(x, y, \delta) \sim\left(x^{\prime}, y^{\prime}, \delta^{\prime}\right)$ if and only if $\delta=\delta^{\prime}$ and $(S x, S y)=\left(x^{\prime}, y^{\prime}\right)$ for some $S \in S O_{\delta}\left(\mathbb{R}^{3}\right)$. 
Composing the maps $\Lambda_{\pi}$ and $\Psi$ we obtain

Proposition 11. The map $\Lambda_{\pi} \Psi: \mathcal{K}_{3} \rightarrow \mathcal{A}_{4}^{q 0}$, given by $\Lambda_{\pi} \Psi(x, y, \delta)=\mathbb{R} \times \mathbb{R}^{3}$ with multiplication

$$
(\alpha, v)(\beta, w)=\left(\alpha \beta-v^{T} w+v^{T} M_{x} w, \alpha w+\beta v+M_{y} \pi(v \wedge w)+\Delta_{\delta} \pi(v \wedge w)\right),
$$

induces a bijection $\bar{\Lambda}_{\pi} \bar{\Psi}: \mathcal{K}_{3} / \sim \stackrel{\sim}{\rightarrow} \mathcal{A}_{4}^{q 0} / \simeq$.

Note that any triple $(x, y, \delta) \in \mathcal{K}_{3}$ may be interpreted as a configuration in $\mathbb{R}^{3}$ formed by a pair of points $(x, y)$ and an ellipsoid $E_{\delta}=\left\{z \in \mathbb{R}^{3} \mid z^{T} \Delta_{\delta} z=1\right\}$. In this view, the equivalence of triples $(x, y, \delta) \sim\left(x^{\prime}, y^{\prime}, \delta^{\prime}\right)$ means geometrically that $E_{\delta}=E_{\delta^{\prime}}$ and $(x, y),\left(x^{\prime}, y^{\prime}\right)$ lie in the same orbit under the action of the special orthogonal symmetry group of $E_{\delta}$. Observe moreover that the particular configuration $\left(0,0, \mathbb{S}^{2}\right)$ produces the particular division algebra $\Lambda_{\pi} \Psi(0,0,(1,1,1))=$ $\mathbb{H}$.

By Proposition 11, any cross-section $\mathcal{C}$ for $\mathcal{K}_{3} / \sim$ classifies all 4-dimensional real quadratic division algebras in the sense that $\Lambda_{\pi} \Psi(\mathcal{C})$ is a cross-section for $\mathcal{A}_{4}^{q 0} / \simeq$. Due to the geometric interpretation of $\mathcal{K}_{3}$, cross-sections $\mathcal{C}$ for $\mathcal{K}_{3} / \sim$ can be detected through 'inspection by the eye'. One possible choice for $\mathcal{C}$ is published in [4. It contains the 9-parameter family of pairwise nonequivalent configurations $(x, y, \delta)$, where $0<\delta_{1}<\delta_{2}<\delta_{3}, x_{1}>0$ and $x_{2}>0$.

In case $m=7$, the question whether the image of $\Lambda_{\pi}: \mathcal{P}_{7} \rightarrow \mathcal{A}_{8}^{q 0}$ exhausts all isoclasses of $\mathcal{A}_{8}^{q 0}$ is still open. It is equivalent to the interesting question whether any dissident morphism $\eta: \mathbb{R}^{7} \wedge \mathbb{R}^{7} \rightarrow \mathbb{R}^{7}$ admits a factorization $\eta=\varepsilon \pi$ into a vectorproduct $\pi: \mathbb{R}^{7} \wedge \mathbb{R}^{7} \rightarrow \mathbb{R}^{7}$ and a definite endomorphism $\varepsilon: V \rightarrow V$. However, irrespective of the answer to this question we may use the map $\Lambda_{\pi}: \mathcal{P}_{7} \rightarrow \mathcal{A}_{8}^{q 0}$ to construct large families of 8 -dimensional quadratic division algebras.

Proposition 12. Let $\mathcal{K}_{7}^{<}=\left\{(X, Y, \delta) \in \mathbb{R}_{\text {ant }}^{7 \times 7} \times \mathbb{R}_{\text {ant }}^{7 \times 7} \times \mathcal{T}_{7} \mid 0<Y_{i j}\right.$ for all $1 \leq$ $i<j \leq 7$ and $\left.0<\delta_{1}<\ldots<\delta_{7}\right\}$, and define $\Psi: \mathcal{K}_{7}^{<} \rightarrow \mathcal{P}_{7}$ by $\Psi(X, Y, \delta)=$ $\left(X, Y+\Delta_{\delta}\right)$. Then $\Lambda_{\pi} \Psi\left(\mathcal{K}_{7}^{<}\right)$is a 49-parameter family of pairwise nonisomorphic objects in $\mathcal{A}_{8}^{q 0}$. The composed map $\Lambda_{\pi} \Psi: \mathcal{K}_{7}^{<} \rightarrow \mathcal{A}_{8}^{q 0}$ is given explicitly by $\Lambda_{\pi} \Psi(X, Y, \delta)=\mathbb{R} \times \mathbb{R}^{7}$, with multiplication

$$
(\alpha, v)(\beta, w)=\left(\alpha \beta-v^{T} w+v^{T} X w, \alpha w+\beta v+Y \pi(v \wedge w)+\Delta_{\delta} \pi(v \wedge w)\right) .
$$

Proof. Let $(X, Y, \delta),\left(X^{\prime}, Y^{\prime}, \delta^{\prime}\right) \in \mathcal{K}_{7}^{<}$such that $\Lambda_{\pi} \Psi(X, Y, \delta) \sim \Lambda_{\pi} \Psi\left(X^{\prime}, Y^{\prime}, \delta^{\prime}\right)$. Then $\left(S X S^{T}, S Y S^{T}, S \Delta_{\delta} S^{T}\right)=\left(X^{\prime}, Y^{\prime}, \Delta_{\delta^{\prime}}\right)$ for some $S \in O_{\pi}\left(\mathbb{R}^{7}\right)$, by Theorem 8 (iii). From $S \Delta_{\delta} S^{T}=\Delta_{\delta^{\prime}}$ we deduce that $\delta=\delta^{\prime}$ and $S=\Delta_{\epsilon}$ for some $\epsilon \in\{1,-1\}^{7}$. From $S Y S^{T}=Y^{\prime}$ we deduce further that $S=\mathbb{I}_{7}$ or $S=-\mathbb{I}_{7}$. Hence $(X, Y, \delta)=$ $\left(X^{\prime}, Y^{\prime}, \delta^{\prime}\right)$.

\section{Historical note}

The problem of constructing or even classifying finite-dimensional real division algebras is as old as the discovery of the quaternion algebra $\mathbb{H}$ (Hamilton 1843) and of the octonion algebra $\mathbb{( 1 )}$ (Graves 1843, Cayley 1845). Frobenius' theorem, asserting that $\{\mathbb{R}, \mathbb{C}, \mathbb{H}\}$ classifies all associative finite-dimensional real division algebras, appeared in 1878 (9]). In 1931, Zorn generalized it to the statement that $\{\mathbb{R}, \mathbb{C}, \mathbb{H}, \mathbb{O}\}$ classifies all alternative finite-dimensional real division algebras ([15]). A natural further generalization to ask for is the classification of all powerassociative finite-dimensional real division algebras. Due to the famous $(1,2,4,8)$ Theorem ([10],[13], [2], [1]) it suffices to classify these in dimensions 4 and 8. 
In fact we have achieved their classification in dimension 4 , and we have constructed a 49-parameter family of them in dimension 8. Namely by [4], Lemma 3 , a finite-dimensional real division algebra is power-associative if and only if it is quadratic.

\section{REFERENCES}

1. J. F. Adams: Vector fields on spheres. Ann. of Math. 75, 603-632 (1962). MR 25:2614

2. M. F. Atiyah and F. Hirzebruch: Bott periodicity and the parallelizability of the spheres. Proc. Cambridge Phil. Soc. 57, 223-226 (1961). MR 23:A3578

3. L. E. Dickson: Linear algebras with associativity not assumed. Duke Math. J. 1 (1935), 113125. MR 22:9516

4. E. Dieterich: Zur Klassifikation vierdimensionaler reeller Divisionsalgebren. Math. Nachr. 194 (1998), 13-22. MR 99f:17001

5. E. Dieterich: Power-associative real division algebras. CMS Conference Proceedings Vol. 24 (1998), 139-144. MR 99j:17002

6. E. Dieterich: Real quadratic division algebras. To appear in Communications in Algebra.

7. E. Dieterich: Dissident Algebras. To appear in Colloquium Mathematicum.

8. B. Eckmann: Stetige Lösungen linearer Gleichungssysteme. Comm. Math. Helv. 15, 318-339 (1942/43). MR 5:104h

9. F. G. Frobenius: Über lineare Substitutionen und bilineare Formen. Journal für die reine und angewandte Mathematik 84, 1-63 (1878).

10. H. Hopf: Ein topologischer Beitrag zur reellen Algebra. Comment. Math. Helv. 13, 219-239 (1940/41). MR 3:61c

11. M. Koecher and R. Remmert: Isomorphiesätze von Frobenius, Hopf und Gelfand-Mazur. Zahlen, Springer-Lehrbuch, 3. Auflage, 182-204 (1992).

12. M. Koecher and R. Remmert: Kompositionsalgebren. Satz von Hurwitz. VektorproduktAlgebren. Zahlen, Springer-Lehrbuch, 3. Auflage, 219-232 (1992).

13. J. Milnor: Some consequences of a theorem of Bott. Ann. of Math. 68, 444-449 (1958). MR 21:1591

14. J. M. Osborn: Quadratic division algebras. Trans. Amer. Math. Soc. 105, 202-221 (1962). MR 25:3968

15. M. Zorn: Theorie der alternativen Ringe. Abh. Math. Sem. Hamburg 8 (1931),123-147.

Uppsala Universitet, Matematiska Institutionen, Box 480, S-751 06 Uppsala, Sverige

E-mail address: Ernst.Dieterich@math.uu.se 